OPEN ACCESS

Edited by:

Adrian Preda,

University of California, Irvine,

United States

Reviewed by:

Błażej Misiak,

Wrocław Medical University, Poland

Raquel Romay-Tallon,

University of Illinois at Chicago,

United States

*Correspondence:

HuaLin Cai

hualincai@csu.edu.cn

Jeffrey K. Yao

jkyao@pitt.edu

Specialty section:

This article was submitted to

Neuropharmacology,

a section of the journal

Frontiers in Neuroscience

Received: 12 December 2018

Accepted: 03 July 2020

Published: 29 July 2020

Citation:

Zhou X, Long T, Haas GL, Cai H and Yao JK (2020) Reduced Levels and Disrupted Biosynthesis Pathways

of Plasma Free Fatty Acids

in First-Episode Antipsychotic-Naive

Schizophrenia Patients.

Front. Neurosci. 14:784.

doi: 10.3389/fnins.2020.00784

\section{Reduced Levels and Disrupted Biosynthesis Pathways of Plasma Free Fatty Acids in First-Episode Antipsychotic-Naïve Schizophrenia Patients}

\author{
Xiang Zhou 1,2, Tao Long 1,2, Gretchen L. Haas ${ }^{2,3}$, HuaLin Cai ${ }^{4,5 *}$ and Jeffrey K. Yao 1,2,3* \\ 'Department of Pharmaceutical Sciences, University of Pittsburgh School of Pharmacy, Pittsburgh, PA, United States, \\ ${ }^{2}$ Medical Research Service and The VISN 4 Mental Illness Research, Education, and Clinical Center, VA Pittsburgh \\ Healthcare System, Pittsburgh, PA, United States, ${ }^{3}$ Department of Psychiatry, University of Pittsburgh School of Medicine, \\ Pittsburgh, PA, United States, ${ }^{4}$ The Department of Pharmacy, The second Xiangya Hospital of Central South University, \\ Changsha, China, ${ }^{5}$ Institute of Clinical Pharmacy, Central South University, Changsha, China
}

Membrane phospholipid deficits have been well-documented in schizophrenia (SZ) patients. Free fatty acids (FFAs) partially come from the hydrolysis of membrane phospholipids and serve as the circulating pool of body fatty acids. These FFAs are involved in many important biochemical reactions such as membrane regeneration, oxidation, and prostaglandin production which may have important implications in SZ pathology. Thus, we compared plasma FFA levels and profiles among healthy controls (HCs), affective psychosis (AP) patients, and first-episode antipsychoticnaive schizophrenia (FEANS) patients. A significant reduction of total FFAs levels was observed in SZ patients. Specifically, significant reductions of 16:0, 18:2n6c, and 20:4n6 levels were detected in FEANS patients but not in APs when compared with levels in HCs. Also, disrupted metabolism of fatty acids especially in saturated and n-6 fatty acid families were observed by comparing correlations between precursor and product fatty acid levels within each fatty acid family. These findings may suggest an increased demand of membrane regeneration, a homeostatic imbalance of fatty acid biosynthesis pathway and a potential indication of increased beta oxidation. Collectively, these findings could help us better understand the lipid metabolism with regard to SZ pathophysiology.

Keywords: first-episode schizophrenia, free fatty acids, antipsychotic-naïve, fatty acid pathway, plasma

\section{INTRODUCTION}

Schizophrenia (SZ) is a devastating neuropsychiatric disorder that affects approximately 20 million people worldwide (GBD 2017 Disease and Injury Incidence and Prevalence Collaborators, 2018). It imposes a massive economic burden on individuals with SZ, their families and the society. Previous studies have linked SZ to widespread structural and functional brain alterations, including multiple neurotransmitter pathway disruptions (Walsh et al., 2008; Kerner, 2009; Karam et al., 2010; Brisch et al., 2014), white matter changes (Kubicki et al., 2005; Lener et al., 2014), and prefrontal-limbic 
network dysfunctions (Weinberger et al., 1988, 1992). Despite plenty of theories proposed on the pathophysiology of SZ, none of them can fully explain its symptomatology and underlying etiology. Given the diverse biological findings reported in SZ, it is possible that the etiologic heterogeneity of SZ could result from a common pathogenic pathway (or a few pathways) that eventually leads to the clinical syndromes.

As far as we know, currently no literature data illustrating whether direct correlations between levels of plasma free fatty acids (FFAs) and the composition of membrane FAs has been reported. However, there is some evidence from two aspects which suggests indirect associations between them. Firstly, plasma FFAs have long been considered as a pivotal indicator of essential FAs status of humans, reflecting the amount of FA source that can be utilized in membrane phospholipid metabolism (Holman et al., 1979). In support, a study has reported about the FA composition of plasma and erythrocyte membranes in normal individuals (Manku et al., 1983). It shows that most ratios of FAs in plasma are like those in erythrocyte membranes except for 18:2n-6 and 22:4n-6, thought the phenomenon was not discussed. Secondly, in vitro, the cellular FA composition is likely to change due to the altered FA levels in serum used to grow established cell lines (Stoll and Spector, 1984). In vivo, supplementation of cod fish oil which is rich in omega-3 polyunsaturated fatty acids (PUFA), will result in increased eicosapentanoic and docosahexaenoic acid incorporations into the total phospholipids of plasma, platelets, and erythrocytes in a dose- and timedependent manner (Von Schacky and Weber, 1985). Moreover, it is well-established that phospholipase $\mathrm{A}_{2}\left(\mathrm{PLA}_{2}\right)$ can recognize and cleave FAs from membrane phospholipids. It can bind to the sn-2 acyl bond of phospholipids and catalytically hydrolyze it, releasing arachidonic acid and lysophosphatidic acid into the blood stream (Dennis, 1994). These may suggest that plasma FA and membrane FA composition are dynamically linked.

As a major class of components of myelin, phospholipids play a crucial role in maintaining myelinated neuronal axons in the central nervous system (CNS) and peripheral nervous system (PNS). Studies show that at least $70 \%$ of the dry mass of both CNS and PNS myelin is formed by phospholipids (Baumann and Pham-Dinh, 2001; Chrast et al., 2010). Interestingly, previous findings (Gattaz et al., 1990; Horrobin et al., 1991; Pettegrew et al., 1991; Noponen et al., 1993) have indicated that in both CNS and peripheral tissues, SZ patients had an increased breakdown of membrane phospholipids due to $\mathrm{PLA}_{2}$ activity. Recent studies have shown that calcium-independent $\mathrm{PLA}_{2}$ plays a crucial role in the regulation of phospholipid metabolism and cell membranes functionality in the CNS (St-Gelais et al., 2004; Schaeffer et al., 2005). In addition, increased activities PLA 2 in SZ patients including drug-naïve patients, drug-free patients and patients with different stages of SZ have been observed (Smesny et al., 2005; Šakić et al., 2016). It is also reported that higher concentrations of $\mathrm{PLA}_{2}$ in those patients are positively correlated with the illness duration and episode numbers (Šakić et al., 2016), while other studies suggest significant correlations between PLA $_{2}$ activity and positive symptoms (Gattaz et al., 1990; Ross et al., 1997). The resulting FFAs, which are not bound on cell membranes or proteins, are involved in many important biochemical reactions such as regeneration of membrane phospholipids (Rapoport, 2001; Yehuda et al., 2002), production of prostaglandins (Flower and Blackwell, 1976; Kuehl and Egan, 1980) and generation of adenosine triphosphates via beta-oxidation in the mitochondria. FFAs originate from lipolysis of membrane phospholipids and serve as dynamic lipid pools in the body. Therefore, changes of FFA levels may have significant implications in the SZ pathology. While lots of studies focused on membrane phospholipid abnormalities, no study has investigated the potential role of plasmatic FFAs in SZ pathology. The aim of the current study thus is to test whether levels and biosynthesis of plasma FFAs are altered in SZ patients at early stage of disease development. Levels of FFAs were measured and compared between the HC group and first-episode antipsychoticnaïve schizophrenia (FEANS) group. To test if those potential alterations of FFA profiles were unique features on SZ patients and could be served as potential biomarkers of SZ, the affective psychosis (AP) group including patients with bipolar disorder and major depression was further added to the comparisons of FFAs levels with the HC and FEANS groups. Furthermore, correlations between the levels of different types of fatty acids were explored in distinct groups of patients and controls.

\section{MATERIALS AND METHODS}

\section{Clinical Design}

Forty SZ antipsychotic-naïve patients were recruited during their first episode of psychosis after they provisionally met diagnostic and statistical manual of mental disorders (DSM-IV) criteria for schizophrenia or schizophreniform disorder based on the Structured Clinical Interview for DSM Disorders. In addition, 52 age-, race-, BMI-, and gender-matched healthy control (HC) subjects and 24 patients with other psychotic disorders (including psychotic bipolar disorder and major depression with psychotic features), herein referred to as AP subjects, were also recruited from the same community. Subjects in AP group were in drug-free status at the time of enrollment. Statistical Manual of Mental Disorders (DSM) version 4 was used to diagnose the patients. To be diagnosed with SZ, two of the following characteristic symptoms need to be met over at least 1 month: delusions, hallucinations, disorganized speech, grossly disorganized or catatonic behavior or negative symptoms (e.g., flattened affect, alogia, amotivation, and avolition). Also, the person should have social or occupational dysfunction or failure to achieve a level of functioning expected for their age and socioeconomic background. Lastly, continuous signs of the disturbance must persist for a minimum of 6 months. No somatic and psychiatric comorbidities were recorded on those SZ patients. None of subjects were taking alcohol or cigarettes for at least 14 days prior to the study or during the study. Also, none of the subjects were on anticoagulants or lipid lowering agents before or during the study. We confirmed that all participants did not have an unbalanced diet, restricted diet or abnormal eating habits. Eating habits between patients and HCs were basically similar. In addition, blood samples were collected after overnight fasting protocol to minimize the diet effect as well. 
Collected fresh blood was immediately separated for plasma and transferred to the $-70^{\circ} \mathrm{C}$ freezer. Clinical symptoms were evaluated prior to initiation of clinicians' choice of antipsychotic agents. Scale for the assessment of positive symptoms (SAPS) and scale for the assessment of negative symptoms (SANS) were used with the FEANS and AP group evaluation. The SAPS has 34 items (including four global items) that constitute four subscales measuring hallucinations, delusions, bizarre behavior, and positive formal thought disorder. The SANS has 25 items (including five global items) that constitutes five subscales measuring affective flattening or blunting, alogia, avolitionapathy, anhedonia-asociality, and attention. Each item was scored by clinicians from 0 (no symptom) to 5 (most severe) to evaluate the degree of each symptom. The demographic and clinical characteristics of subjects are shown in Table $\mathbf{1 .}$

\section{Clinical Ethics}

The study was approved by the Institutional Review Boards of the University of Pittsburgh and the VA Pittsburgh Healthcare System. All patients and control subjects were recruited from UPMC Western Psychiatric Hospital in Pittsburgh. Diagnostic assessments and clinical symptom ratings were performed by experienced research clinicians, then reviewed and confirmed by senior diagnosticians during diagnostic conferences attended by research faculty experienced psychiatrists. All subjects including controls were provided written informed consent prior to participation in the research procedures.

\section{Fatty Acid Methylation and Gas Chromatography Analysis}

Plasma FFAs were quantitatively determined by capillary gas chromatography (GC) according to the procedure described by Lepage and Roy (1988). In brief, FFAs were extracted from $50 \mu \mathrm{l}$ of plasma containing internal standard (Tridecanoic acid) and then converted to methyl esters by acetyl chloridemethanol reagent. After the preparation, $1 \mu l$ of the resulting fatty acid methyl esters was injected into GC for analysis. The procedure used for GC analysis has been previously described (Yao et al., 2002; Reddy et al., 2004). Briefly, prepared samples including internal standard were injected into the capillary column, with programmed control of oven temperatures. Each sample was run under a splitless injection mode with helium as the carrier gas ( $3 \mathrm{~mL} /$ minute) and with an inlet pressure of 6.5 psi. All major peaks were eluted within 16 min. Typical chromatography from a HC subject is shown in Supplementary Figure 2. These peaks were then identified and determined by comparing the retention times with those of standard mixtures (Supelco, Inc.). Linear standard curve range of this assay is from $0.25 \mathrm{nmol} / \mathrm{mL}$ to $1250 \mathrm{nmol} / \mathrm{mL}$. The Lower Limit of Quantification is $0.05 \mathrm{nmol} / \mathrm{mL}$. Concentrations of each FFA were then calculated by Agilent ChemStation. Each sample was tested in duplicates and quality control samples made from the plasma pool were used to check the differences between batches. Intra-assay percentage of $\mathrm{CV}$ was below $5.0 \%$ and inter-assay percentage of CV was below $6.5 \%$.

\section{Data Analysis Packages and Procedures}

All tests were done using STATA (v 15.0) software packed. Two-tailed $p$-values were applied to test the fatty acid levels between groups. First, All data were checked for skewness by quantile-quantile plots. Second, for FFA levels comparisons, analysis of variance (ANOVA) with Bonferroni corrections on post hoc comparisons were conducted across the HC, AP, and FEANS groups. Third, Pearson correlation coefficients were used to test associations between precursor FAs and product FAs within certain fatty acid pathways. Fourth, Pearson correlation coefficients were used to test associations between FAs and clinical scales.

As there were significant differences between groups in terms of sex and race (Table 1), these variables were used as covariates in the statistical analysis. ANOVA analysis with Bonferroni corrections on post hoc comparisons were conducted to test differences between FFA levels across the HC, AP, and FEANS groups. The Bonferroni correction was applied when testing multiple null hypothesis in one data set. Therefore, the critical $p$-value was adjusted to the number of comparisons in a specific ANOVA post hoc test. Since FFAs levels among three groups (three comparisons) were compared, the critical $p$-value was set to: $0.05 / 3=0.0167$. Pearson correlation coefficients were used to test associations between precursor FAs and product FAs within certain fatty acid pathways. These precursor/product FFAs correlation analyses were run with sex and race as covariates. These correlations were utilized to reflect connections between the precursor and product FFAs. We hypothesize that in SZ patients, a disturbance of FFAs pathways will lead to the loss of tight connections between precursor and product FFAs in comparison to those in control groups. Also, correlations between precursor and product FAs can indirectly implicated the activity of the elongase and desaturase as indicated in Supplementary Figure 1. Therefore, Correlation analysis were conducted among major FAs within each FA family (n-6, n$7, \mathrm{n}-9, \mathrm{n}-3$, and saturated fatty acids) pathway. Specifically, $18: 2 \mathrm{n} 6,18: 3 \mathrm{n} 6,20: 3 \mathrm{n} 6,20: 4 \mathrm{n} 6$, and $22: 4 \mathrm{n} 6$ were included in $\mathrm{n}-6$ correlation analyses; $16: 1 \mathrm{n} 7 \mathrm{t}, 16: 1 \mathrm{n} 7 \mathrm{c}$, and $18: 1 \mathrm{n} 7$ were included in $\mathrm{n}-7$ correlation analyses; $18: 1 \mathrm{n} 9 \mathrm{t}$ and $18: 1 \mathrm{n} 9 \mathrm{c}$ were included in $n-9$ correlation analyses; $22: 5 n 3$ and 22:6n3 were included in n-3 correlation analyses; 14:0, 15:0, 16:0, $17: 0,18: 0,21: 0,22: 0$, and 17:1 were included in saturated correlation analyses. Bonferroni corrections were applied to the number of comparisons in the correlation analyses. Precursor/product pairs that were analyzed: 18:2n6/18:3n6, $18: 3 n 6 / 20: 3 n 6, \quad 20: 3 n 6 / 20: 4 n 6, \quad 20: 4 n 6 / 22: 4 n 6, \quad 18: 2 n 6 / 20: 3 n 6$, 18:2n6/20:4n6, 18:2n6/22:4n6, 18:3n6/20:4n6, 20:3n6/22:4n6, and $20: 3 n 6 / 22: 4 \mathrm{n} 6$ for the n-6 family (Bonferroni corrected $\alpha$ level $=0.05 / 10=0.005) ; 16: 1 \mathrm{n} 7 \mathrm{t} / 16: 1 \mathrm{n} 7 \mathrm{c}, 16: 1 \mathrm{n} 7 \mathrm{t} / 18: 1 \mathrm{n} 7$, and $16: 1 \mathrm{n} 7 \mathrm{c} / 18: 1 \mathrm{n} 7$ for the $\mathrm{n}-7$ family (Bonferroni corrected $\alpha$ level $=0.05 / 3=0.0167) ; 18: \ln 9 \mathrm{t} / 18: 1 \mathrm{n} 9 \mathrm{c}$ for the $\mathrm{n}-9$ family $(\alpha$ level $=0.05) ; 22: 5 \mathrm{n} 3 / 22: 6 \mathrm{n} 3$ for the $n-3$ family $(\alpha$ level $=0.05) ; 14: 0 / 16: 0,16: 0 / 18: 0,18: 0 / 22: 0,14: 0 / 18: 0$, $14: 0 / 22: 0,16: 0 / 22: 0,15: 0 / 17: 0$, and 17:0/17:1 for the saturated fatty acids (Bonferroni corrected $\alpha$ level $=0.05 / 8=0.0063$ ). For the significant correlations that found between n-3 FFAs 
TABLE 1 | Demographic data of recruited subjects.

\begin{tabular}{|c|c|c|c|c|c|c|}
\hline & \multirow[t]{2}{*}{$\mathrm{HC}$} & \multirow[t]{2}{*}{ AP } & \multirow[t]{2}{*}{ FEANS } & \multicolumn{3}{|c|}{ Comparisons } \\
\hline & & & & HC vs. AP & HC vs. FEANS & AP vs. FEANS \\
\hline Numbers & 52 & 24 & 40 & NA & NA & NA \\
\hline Sex ${ }^{a}$ & $31 / 20$ & $13 / 11$ & $26 / 14$ & $<0.001$ & $<0.001$ & $<0.001$ \\
\hline $\mathrm{Age}^{1}$ & $26.3 \pm 7.4$ & $26.9 \pm 9.9$ & $22.7 \pm 8.1$ & ns & ns & ns \\
\hline $\mathrm{BMI}^{2}$ & $25.2 \pm 4.4$ & $25.2 \pm 6.9$ & $23.3 \pm 5.3$ & ns & ns & ns \\
\hline Race $^{b}$ & $34 / 10 / 3 / 4$ & 17/5/1/1 & $20 / 16 / 2 / 2$ & $<0.001$ & $<0.001$ & $<0.001$ \\
\hline SAPS $^{3}$ & NA & $18.2 \pm 14.9$ & $26.0 \pm 12.2$ & NA & NA & 0.0265 \\
\hline SANS $^{4}$ & NA & $37.6 \pm 9.5$ & $44.1 \pm 11.1$ & NA & NA & 0.0202 \\
\hline
\end{tabular}

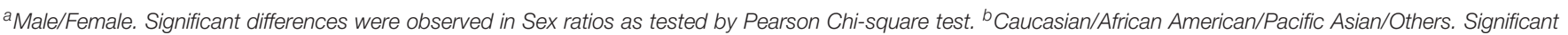

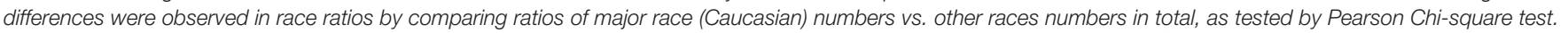

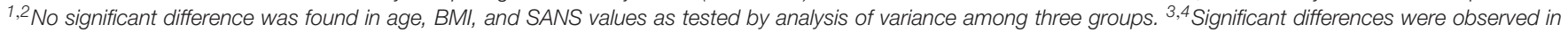

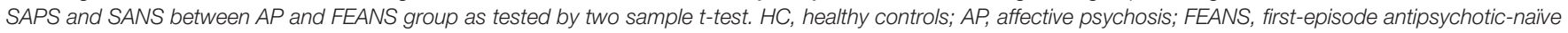

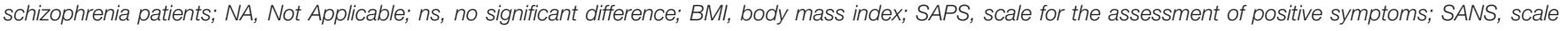

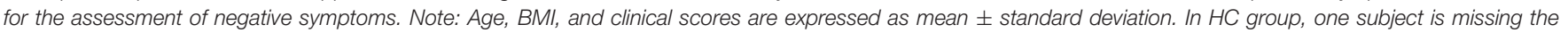
information of sex and race. Three subjects in FEANS group and one subject in AP group are missing BMI data due to missing height/weight values.

levels and clinical scales, the corrected Bonferroni corrected $\alpha$ level $=0.05 / 4=0.0125$ (SAPS/22:5n3, SAPS /22:6n , SANS/22:5n3, and SANS/22:6n3).

\section{RESULTS}

\section{Comparisons of Plasma Fatty Acids Levels Among All Groups}

Significantly lower total levels of plasma FFAs were detected in FEANS subjects than in HC and AP subjects (Table 2). Specifically, the reductions of plasma FFAs were mostly noted in saturated and n-6 fatty acid families (Table 2). Among saturated fatty acids, the 16:0 levels were significantly decreased in the FEANS group as compared with those in the HC and AP groups (Table 2). Among the n-6 family of fatty acids, the 18:2n6c and 20:4n6 levels were significantly lower in the FEANS group as compared with the $\mathrm{HC}$ and AP groups (Table 2). On the other hand, no significant differences were observed in each of these FFA levels between $\mathrm{HC}$ and AP groups. Among the n-3 family of fatty acids, significantly lower total levels of n-3 FFAs were observed in the FEANS group as compared with the HC group. However, no significant differences in n-3 FFA levels were detected between the FEANS group and the AP group compared to the n-3 FFAs.

\section{Correlations Within Fatty Acid Families in HC, AP, and FEANS Groups}

There was no significant correlation between any covariate (sex/age) and any FFA index. In the present study, correlations between fatty acids within each fatty acid family were also examined with sex and age as covariates. As expected, there were significant correlations between product and precursor within certain fatty acid families, among the HC, AP, and FEANS groups. Specifically, significant correlations were detected mainly in saturated fatty acids, between 16:0 and 18:0, 16:0 and 22:0, and between 18:0 and 22:0 (Figure 1). For the other families of fatty acids, however, significant correlations were only observed in one or two of the three groups.

In contrast with the findings mentioned above, significant correlations between $18: 3 \mathrm{n} 6$ and $20: 4 \mathrm{n} 6$, as well as between $16: 1 \mathrm{n} 7 \mathrm{c}$ and $18: 1 \mathrm{n} 7$, were identified only in the HC group, but not in FEANS or AP groups (Figure 2). Interestingly, significant correlations between 17:0 and 17:1, as well as between 18:3n6 and 20:3n6, were observed both in HC and AP groups, but not in the FEANS group (Figure 3).

\section{Correlations Between Free Fatty Acid Levels and Clinical Scales in FEANS and AP Groups}

In FEANS and AP groups, no significant correlation was found between SANS scores and total or any single FFA levels. However, we observed a significant reversed correlation between levels of $22: 6 \mathrm{n} 3$ and SAPS scores in the FEANS group when comparing with that in the AP group (Figure 4).

\section{DISCUSSION}

In this study, levels of plasma FFAs were determined in SZ patients experiencing their first episode of schizophrenia, patients with a first episode of other (non-schizophrenia) psychotic disorders and HC subjects. Understanding FFA profiles will help us to better comprehend the altered lipid metabolism in SZ. A recent meta-analysis study has revealed that several polyunsaturated FAs levels were decreased in erythrocyte membranes of SZ patients (Van der Kemp et al., 2012). It has also been well-documented that the increased breakdown of membrane phospholipids is mainly due to elevated $\mathrm{PLA}_{2}$ activities in SZ patients (Smesny et al., 2005), both in peripheral tissues (Gattaz et al., 1990; Noponen et al., 1993) and CNS (Horrobin et al., 1991; Pettegrew et al., 1991). It has been welldocumented that there is an increased breakdown of membrane phospholipids due to elevated $\mathrm{PLA}_{2}$ activity in SZ patients, both in peripheral tissues (Gattaz et al., 1990; Noponen et al., 
TABLE 2 | Fatty acid concentrations in healthy controls (HCs, $n=52$ ), affective psychosis (AP, $n=24$ ), and first-episode antipsychotic-naïve schizophrenia patients (FEANS, $n=40)$.

\begin{tabular}{|c|c|c|c|c|c|c|c|}
\hline \multirow[t]{2}{*}{ Fatty acid $^{a}$} & \multirow[t]{2}{*}{$\mathrm{HC}$} & \multirow[t]{2}{*}{ AP } & \multirow[t]{2}{*}{ FEANS } & \multicolumn{4}{|c|}{ Comparisons } \\
\hline & & & & $F^{b}$ & HC vs. AP & HC vs. FEANS & AP vs. FENAS \\
\hline \multicolumn{8}{|l|}{ Saturates } \\
\hline $14: 0$ & $35 \pm 16$ & $29 \pm 12$ & $34 \pm 20$ & 1.065 & ns & ns & ns \\
\hline 15:0 & $4.5 \pm 1.8$ & $5.9 \pm 1.6$ & $3.9 \pm 1.9$ & 9.313 & 0.002 & ns & $<0.0001$ \\
\hline $16: 0$ & $357 \pm 95$ & $356 \pm 82$ & $295 \pm 74$ & 6.889 & ns & 0.0007 & 0.007 \\
\hline $17: 0$ & $6.6 \pm 4.5$ & $6.6 \pm 2.7$ & $4.9 \pm 1.8$ & 3.001 & ns & $\mathrm{ns}$ & ns \\
\hline 18:0 & $109 \pm 30$ & $125 \pm 28$ & $98 \pm 21$ & 7.917 & ns & ns & 0.0004 \\
\hline 21:0 & $5.1 \pm 3.6$ & $6.5 \pm 2.5$ & $4.5 \pm 3.6$ & 2.615 & ns & ns & ns \\
\hline $22: 0$ & $11.2 \pm 4.7$ & $12.6 \pm 4.5$ & $9.0 \pm 3.2$ & 6.160 & ns & ns & 0.001 \\
\hline \multicolumn{8}{|l|}{ Monoenes } \\
\hline $16: 1 n 7 t$ & $5.8 \pm 2.4$ & $6.9 \pm 2.3$ & $4.5 \pm 1.6$ & 9.963 & $\mathrm{~ns}$ & ns & $<0.0001$ \\
\hline $16: 1 n 7 c$ & $22.6 \pm 10.7$ & $26.7 \pm 11.2$ & $17.1 \pm 7.8$ & 7.577 & ns & $\mathrm{ns}$ & 0.0003 \\
\hline $17: 1$ & $9.2 \pm 7.0$ & $12.2 \pm 4.1$ & $7.8 \pm 5.3$ & 4.053 & ns & ns & 0.014 \\
\hline $18: 1 n 9 t$ & $25 \pm 14$ & $27 \pm 11$ & $21 \pm 12$ & 1.864 & $\mathrm{~ns}$ & ns & ns \\
\hline $18: 1 n 9 c$ & $227 \pm 73$ & $213 \pm 54$ & $181 \pm 46$ & 6.549 & ns & 0.001 & ns \\
\hline $18: 1 n 7$ & $25 \pm 10$ & $28 \pm 8$ & $22 \pm 8$ & 4.304 & ns & ns & 0.006 \\
\hline \multicolumn{8}{|l|}{ Dienes } \\
\hline $18: 2 n 6 c$ & $314 \pm 75$ & $322 \pm 65$ & $255 \pm 62$ & 10.511 & $\mathrm{~ns}$ & $<0.0001$ & $<0.0001$ \\
\hline $20: 2 n 6$ & $5.1 \pm 3.6$ & $5.1 \pm 2.3$ & $3.6 \pm 2.4$ & 3.594 & $\mathrm{~ns}$ & 0.013 & ns \\
\hline \multicolumn{8}{|l|}{ Trienes } \\
\hline $18: 3 n 6$ & $5.6 \pm 2.7$ & $5.3 \pm 1.9$ & $4.6 \pm 2.1$ & 2.445 & ns & ns & ns \\
\hline $20: 3 n 6$ & $5.3 \pm 2.9$ & $5.3 \pm 2.3$ & $4.3 \pm 2.8$ & 1.605 & ns & ns & ns \\
\hline \multicolumn{8}{|l|}{ Tetraenes } \\
\hline $20: 4 n 6$ & $70 \pm 17$ & $75 \pm 10$ & $60 \pm 16$ & 7.748 & $\mathrm{~ns}$ & 0.004 & 0.0004 \\
\hline $22: 4 n 6$ & $13.4 \pm 4.6$ & $15.4 \pm 4.1$ & $12.9 \pm 4.5$ & 2.527 & ns & $\mathrm{ns}$ & ns \\
\hline \multicolumn{8}{|l|}{ Pentanenes } \\
\hline $22: 5 n 3$ & $5.0 \pm 2.6$ & $5.7 \pm 2.2$ & $3.9 \pm 2.7$ & 3.869 & ns & ns & 0.009 \\
\hline \multicolumn{8}{|l|}{ Hexananes } \\
\hline $22: 6 n 3$ & $26 \pm 9$ & $25 \pm 7$ & $22 \pm 3$ & 4.584 & ns & 0.004 & ns \\
\hline \multicolumn{8}{|l|}{ Totals } \\
\hline Saturates & $529 \pm 129$ & $542 \pm 118$ & $450 \pm 91$ & 7.012 & $\mathrm{~ns}$ & 0.001 & 0.002 \\
\hline n-3 fatty acids & $31 \pm 10$ & $31 \pm 8$ & $26 \pm 5$ & 5.756 & ns & 0.006 & ns \\
\hline n-6 fatty acids & $413 \pm 90$ & $428 \pm 65$ & $340 \pm 74$ & 12.509 & ns & $<0.0001$ & $<0.0001$ \\
\hline Grand totals & $1288 \pm 297$ & $1315 \pm 213$ & $1069 \pm 205$ & 10.738 & ns & $<0.0001$ & 0.0003 \\
\hline
\end{tabular}

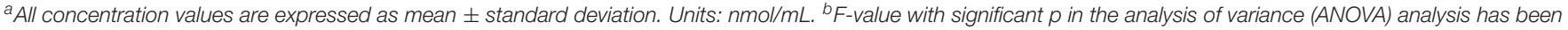
shown in bold. Critical p-values after Bonferroni Correction: 0.0167.

1993) and CNS (Horrobin et al., 1991; Pettegrew et al., 1991). Gattaz and Brunner (1996) proposed that increased PLA 2 activity in the prefrontal cortex could contribute to membrane phospholipid deficits and eventually lead to hypofrontality in schizophrenia. Several later studies supported this theory which linked membrane phospholipid deficits to myelin dysfunctions (Schmitt et al., 2004; Peters et al., 2012) and prefrontal cortex abnormality (Taha et al., 2013). It is hypothesized that increased lipolysis of membrane lipids would also be associated with the dyslipidemia in SZ patients. Both increased levels of serum triglycerides and decreased levels of serum high density lipoproteins were found in antipsychotic-naïve SZ patients, suggesting the disturbance of lipid profiles could occur during the early course of the disease (Misiak et al., 2017). The FFAs released from membrane phospholipids after hydrolysis by PLA enter into the circulating system. Therefore, an increased breakdown of membrane phospholipids through PLA could potentially lead to higher levels of FFAs as we originally hypothesized. However, instead of observing increased FFAs resulting from increased breakdown of membrane phospholipids, we observed significantly lower levels of plasma FFAs and disrupted linkages in FFA biosynthesis pathways among FEANS patients. More importantly, we did not observe significantly reduced FFAs levels in AP group subjects, suggesting such reductions may be trait features of SZ. Since many important biochemical reactions were involved in those FFAs such as regeneration of membrane phospholipids (Rapoport, 2001; Yehuda et al., 2002), production of prostaglandins (Flower and Blackwell, 1976; Kuehl and Egan, 1980) and generation of adenosine triphosphates via beta-oxidation in the mitochondria, significantly lower 

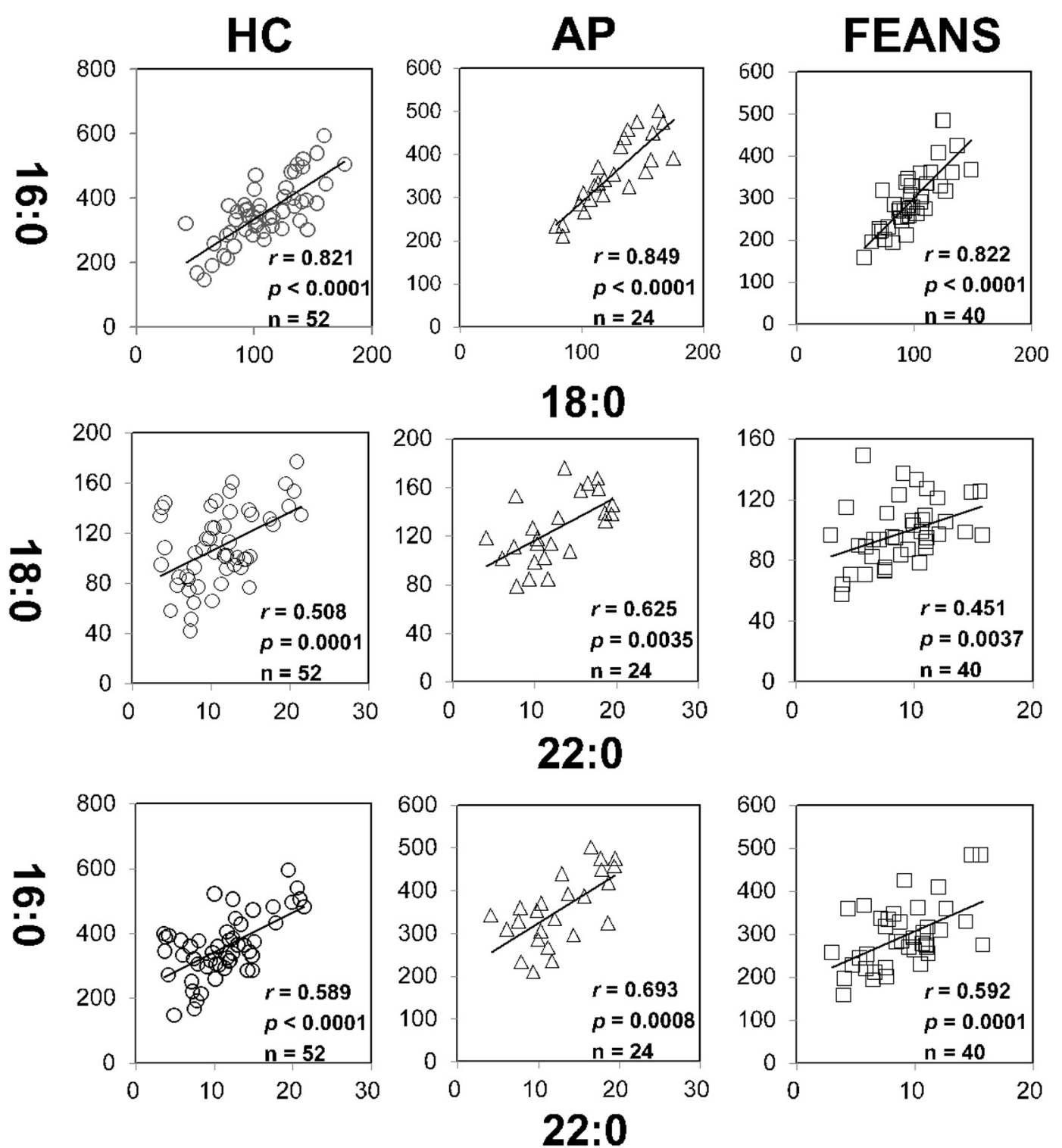

FIGURE 1 | Tight correlations of fatty acids among healthy control (HC) subjects, affective psychosis (AP) patients, and first-episode antipsychotic-naïve schizophrenia patients (FEANS). Critical p-value after Bonferroni correction: 0.0063.

levels of plasma FFAs and breakdown of correlations between precursor/product FAs may serve as biomarkers of SZ, which lead to three important implications with regard to SZ's pathophysiology.

First, reduced total FFA levels may imply an increased regeneration of cell membranes and possible alterations in beta-oxidations of fatty acid metabolism at the early course of SZ. While plenty of studies have focused on the PUFA levels in SZ (Van der Kemp et al., 2012), few have discussed other FAs and the total FA levels in SZ (McEvoy et al., 2013; Yang et al., 2017). Recently, a decreased total plasma FFA levels was detected in FEANS patients with limited sample sizes (Zhou et al., 2017, 2018). Unfortunately, the portion of plasma FFAs after the hydrolysis by $\mathrm{PLA}_{2}$ from those membrane phospholipids has not yet been explored. In our working hypothesis, reduced levels of plasma FFAs may reflect a depleted pool of those fatty acids resulting from an increased demand of cell membrane regeneration during the early course of SZ development. It is speculated that such demand was due to the membrane phospholipid deficits. As suggested by previous literature, oxidative stress could lead to such deficits through membrane lipid peroxidation in SZ patients (Dietrich-Muszalska and Kontek, 2010; Bitanihirwe and Woo, 2011; Boskovic et al., 2011). Notably, while there is a general trend of decrease among all families of FFAs, we found that $n-6$ and saturated family FFA levels in FEANS group are significantly lower than those in $\mathrm{HC}$ and AP groups. This finding implies that these two fatty acid families are most vulnerable to oxidative stress at 

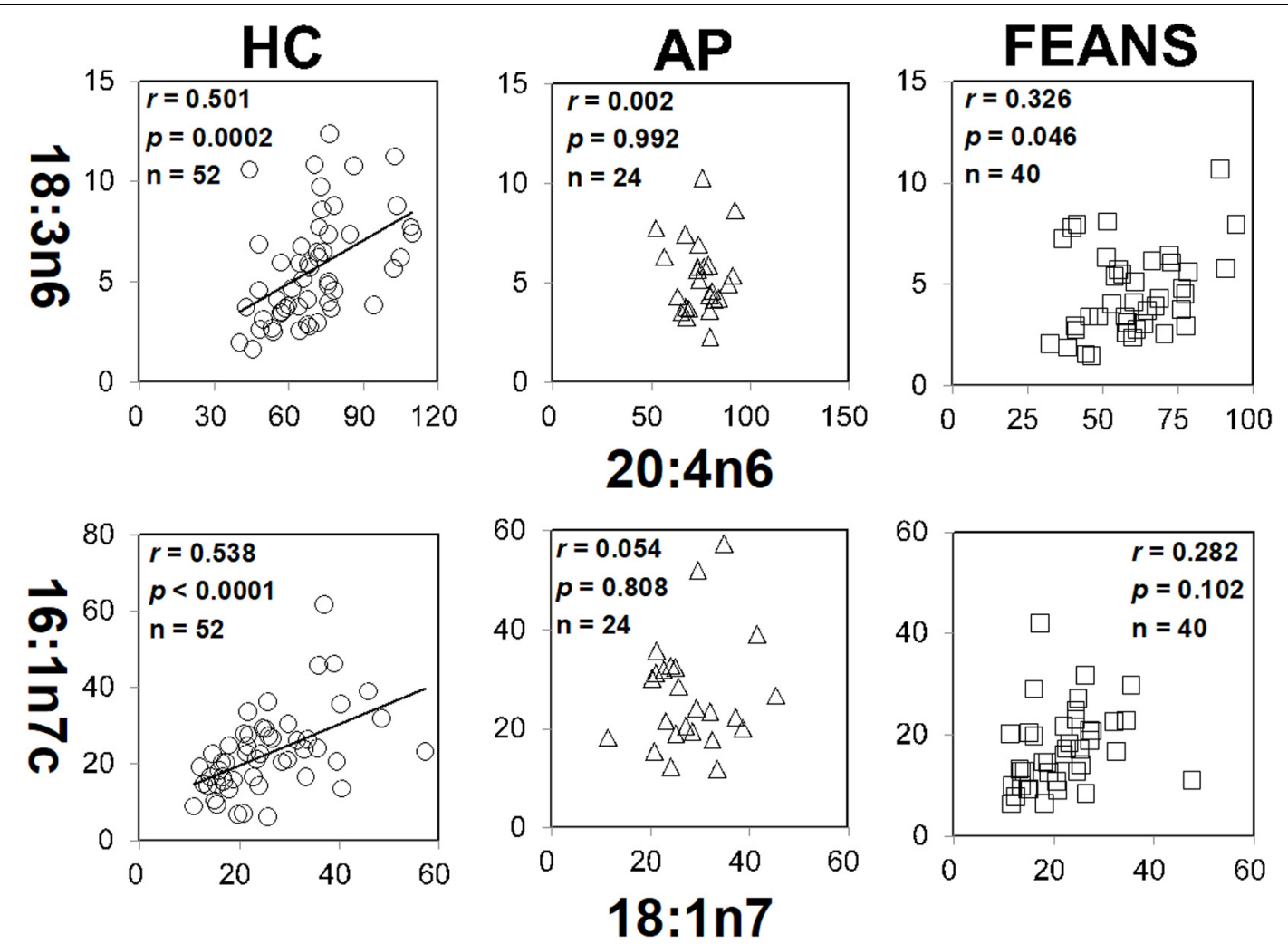

FIGURE 2 | Disrupted correlations of fatty acids both shown in affective psychosis (AP) patients and first-episode antipsychotic-naïve patients (FEANS). Critical $p$-value after Bonferroni correction: for 18:3n6/20:4n6, critical $p=0.005$; for 16:1n7c/18:1n7, critical $p=0.0167$.

the early stage of SZ. As the body cannot synthesize n-6 fatty acids by itself, it might be interesting to discuss the potential dietary effects on these fatty acids here. When the fats get absorbed through digestion system, they will breakdown and then converted to triglycerides and lipoproteins, incorporated into membrane phospholipids and further be broken down into FFAs. Our previous study showed that when comparing membrane-bound fatty acid levels in red blood cell samples, no significant difference in either saturated or monounsaturated fatty acids was observed between FEANS and control groups (Yao et al., 2002). This finding suggested that dietary effects might not have a significant impact on membrane phospholipid levels. In the present study, there was no evidence of metabolic dysfunction observed in these FEANS patients. At the meantime, FEANS subjects' BMI data were comparable with those in controls (Table 1). Therefore, it's not likely that the dietary effects played a significant role here. Furthermore, previous studies showed that several fatty acid beta-oxidation enzymes were significantly increased in the brains of SZ patients, which implied enhanced beta-oxidation in FEANS patients (Prabakaran et al., 2004). Upregulated beta-oxidation in peripheral tissues of SZ patient has also been suggested by comparing product-to-precursor ratios of serum FFAs (Yang et al., 2017). The decreased FFAs levels thus may result from a depleted source in membrane phospholipids as lipids metabolism was shifted toward beta-oxidation in the cytosol. Oxidative stress might play a role in promoting lipid beta-oxidation here, although the impact on FFA metabolism by oxidative stress is still unclear and the mechanism by which it caused the up-regulation of the beta-oxidation remains to be determined. In summary, it is speculated that the reduction of plasma FFA levels may be due to the increased regeneration of membrane phospholipids and hyperactivity of beta-oxidation in SZ pathology. A postulated model is proposed below to explain the reductions of FFA levels (Figure 5) in SZ patients. In brief, the extent of oxidative stress was accumulated in SZ patients due to environmental or genetic factors. The increased oxidative stress levels led to membrane phospholipid deficits, and thus, more FFAs were taken up for regenerating cell membranes in SZ patients. Also, increased oxidative stress levels resulted in an increased activity of beta-oxidation on FFAs, and thus more FFAs were metabolized through this reaction in SZ patients. As a result, reduced levels of total plasma FFAs were observed in the FEANS group when compared with the $\mathrm{HC}$ and the AP groups.

Second, our findings reflect a homeostatic imbalance of fatty acid biosynthesis at the early stage of SZ. Most FFAs can be synthesized by elongases and desaturases, and then go through beta-oxidation to generate energy (Supplementary Figure 2). Although some of precursor to product correlations persisted across disease and normal status, some appeared to be lost in the FEANS group. Our results suggest that the potential for steady formation of fatty acids is altered early in the course of illness. While alterations were also observed in the saturated 

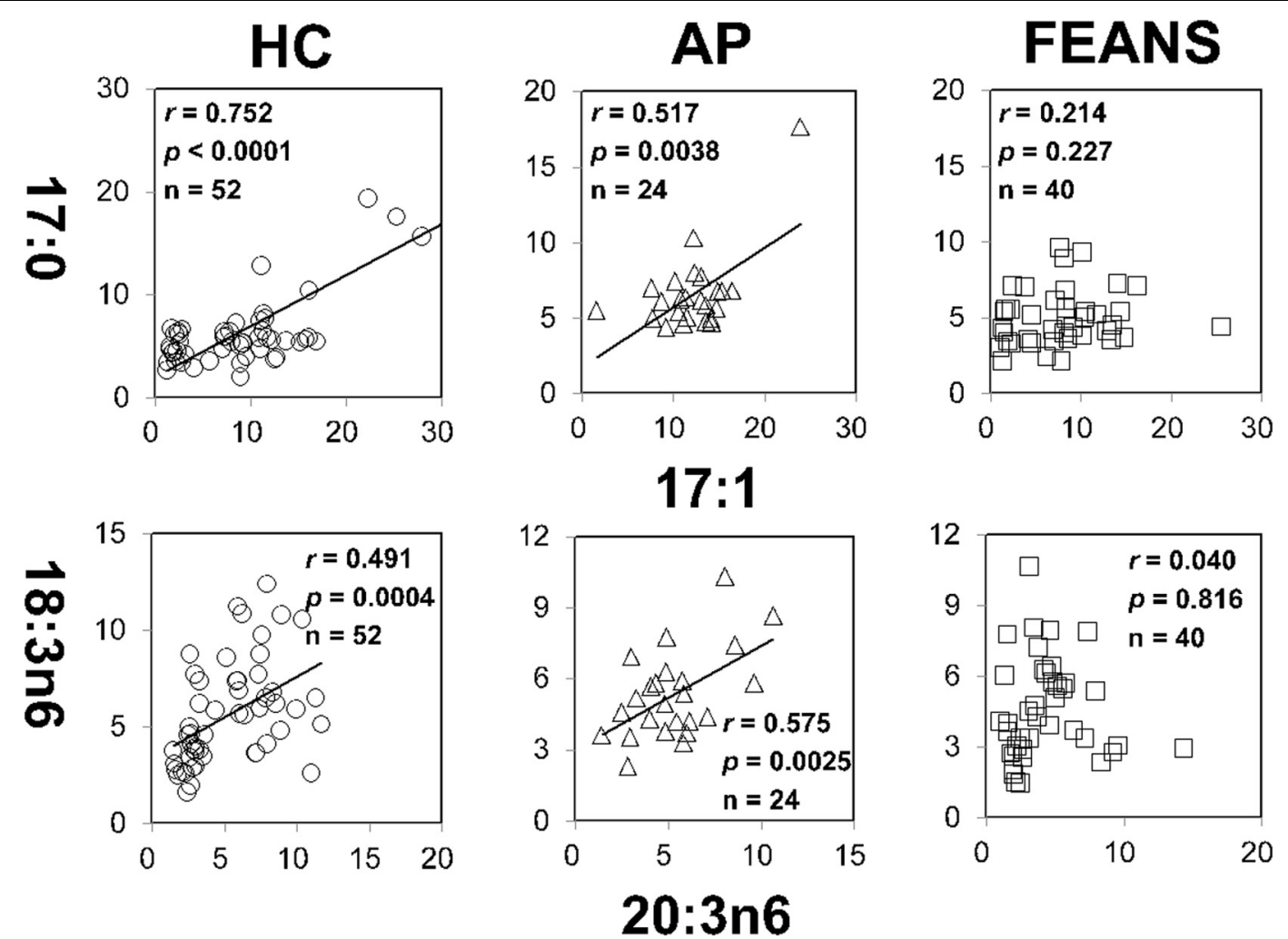

FIGURE 3 | Disrupted correlations of fatty acids only present in first-episode antipsychotic-naiive patients (FEANS). Critical $p$-value after Bonferroni correction: for 17:0/17:1, critical $p=0.0063$; for 18:3n6/20:4n6, critical $p=0.005$.

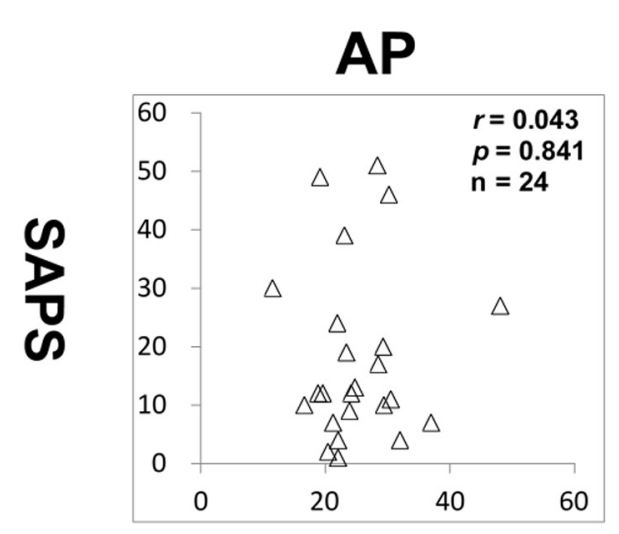

\section{FEANS}

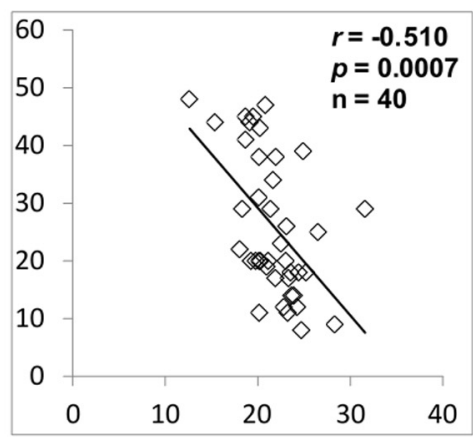

\section{2:6n3}

FIGURE 4 | Significant reversed correlations between 22:6n3 and assessment of positive symptoms (SAPS) in first-episode antipsychotic-naïve patients (FEANS). Critical $p$-value after Bonferroni correction: $p=0.125$.

fatty acid family, it appears that the n- 6 fatty acid family is the one that was most altered in FEANS subjects but not in the AP subjects. This may suggest that the n-6 fatty acid biosynthesis is more disrupted than other fatty acid families at the early course of the SZ while not in the other psychiatric disorders. Although there was no difference of 22:6n 3 between FEANS and
AP groups (Table 2), 22:6n3 levels were negatively associated with SAPS scores only in FEANS and this very correlation is absent in AP group (Figure 4). N-6 PUFA (especially arachidonic acid and palmitic acid) can be viewed as proinflammatory molecules, whereas n-3 PUFA (especially eicosapentaenoic acid and docosahexaenoic acid) can be viewed as anti-inflammatory 

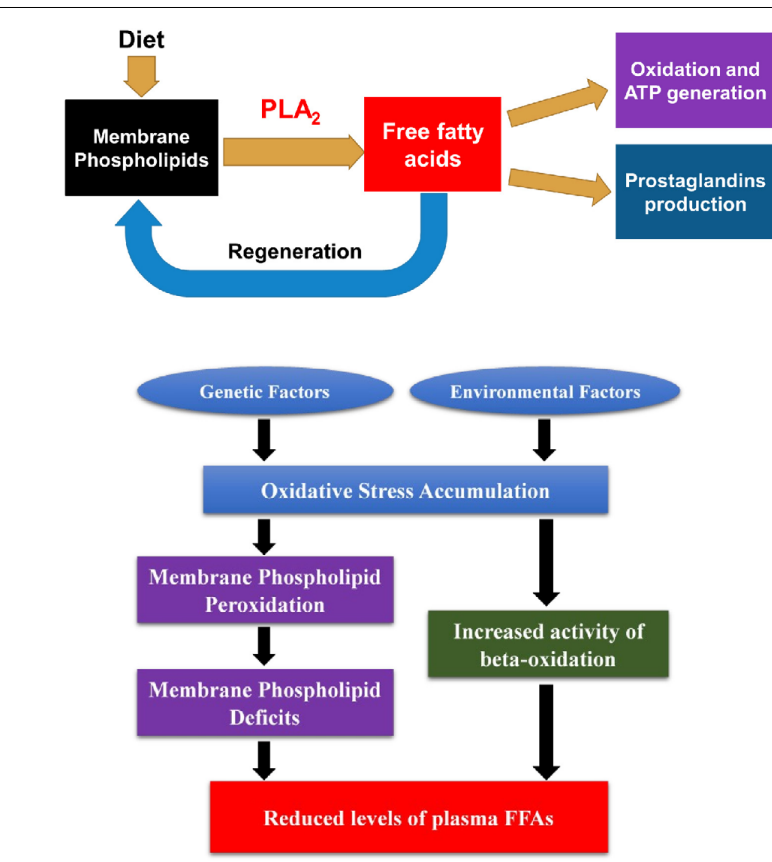

FIGURE 5 | Free fatty acids (FFAs) in lipid metabolism and postulated model of reduced plasma FFA levels and its underlying mechanism in schizophrenia pathophysiology. FFAs come from the lipolysis of membrane phospholipids and serve as an active circulating pool in body. They are involved in many important biochemical reactions including regeneration of membrane phospholipids, beta-oxidation, and production of prostaglandins. In schizophrenia, oxidative stress level was accumulated due to environmental or genetic factors. Consequently, oxidative stress led to membrane phospholipid deficits and increased activity of beta-oxidation of FFAs. Since more FFAs were utilized to regenerate cell membranes and were metabolized through beta-oxidation, levels of plasma FFAs were reduced in SZ patients. PLA ${ }_{2}$, phospholipase $\mathrm{A}_{2}$.

molecules (Sears and Perry, 2015). More disrupted n-6 fatty acid biosynthesis may be considered as a trait-like marker reflecting a severer inflammatory status of SZ patients. However, n-3 PUFA such as 22:6n3 levels could preferably serve as a state-like marker in SZ, which is linked to the symptomatology. However, the exact mechanisms of such alteration on $\mathrm{n}-6$ family FFAs are still unknown. In summary, our findings suggest a disrupted biosynthesis of fatty acids, especially on $\mathrm{n}-6$ fatty acids in the early stage of SZ.

Third, a significantly reduced n-6 FAs may be attributed to the blunted niacin-induced skin flushing in a subgroup of SZ patients, which might be clinically useful in the diagnosis of schizophrenia (Morrow et al., 1989, 1992; Ward et al., 1998; Puri et al., 2001; Benyó et al., 2005; Maciejewski-Lenoir et al., 2006; Tang et al., 2006; Lai et al., 2007; Messamore and Yao, 2016). While the cause of such blunted skin flushing in SZ is still unclear, our findings of a significant decreased arachidonic acid (20:4n6) as well as its precursors in FEANS patients may offer a possible explanation to the underlying mechanism that caused the blunted niacin-induced response in SZ. It is speculated that reduced levels of 18:2n6 and 20:4n6 may reflect a decreased pool for subsequent production of prostaglandin vasodilators $\left(\mathrm{PGD}_{2}\right.$ and
$\mathrm{PGF}_{2}$ ), which could lead to the blunted niacin-induced flushing response in those SZ patients (Messamore et al., 2010).

\section{LIMITATIONS}

The present study of FFAs was mainly focused on FEANS patients, while antipsychotic treatment effects on those FFAs might also be an interesting research target. This is due to the high prevalence of metabolic syndrome among antipsychotic-treated SZ patients, especially among those treated by second-generation antipsychotics (Casey et al., 2004; Wu et al., 2006). Although promising clinical data showed the metabolic improvement of SZ patient by n-3 FAs supplementation in a randomized placebo-controlled trial (Xu et al., 2019), no literature had reported the possible impact of antipsychotics on FFA profiles in terms of metabolic syndrome. A previous preliminary study was conducted by our group to demonstrate a significant increase of total levels of FFAs after risperidone (a second-generation antipsychotic) treatment when compared to baseline FEANS patients (Zhou et al., 2017, 2018). Future in-depth study will be conducted to investigate the effect of antipsychotics on lipid profiles among SZ patients.

Based on our findings, significantly decreased levels of FFAs within $n-6$ family were observed in the FEANS group, which may imply a possible link to the blunted niacin-induced skin flushing in a subgroup of SZ patients. However, niacin response data in this clinical group was not available to validate the potential relationship between $\mathrm{n}-6$ FFAs and niacin-induced skin flushing responses. Further studies are required to correlate plasma n6 FFAs, especially arachidonic acid, with niacin response data. Also, since only a subgroup of SZ patients are presenting such blunted skin flushing, it would be interesting to see if there is a significant difference between a positive-flushing group and a minimal/non-flushing group in terms of arachidonic acid levels.

Most FFAs are synthesized by elongases and desaturases from substrate FFAs, and then go through beta-oxidation to generate energy. However, this study did not measure the activity of elongase and desaturase in those clinical subjects. Instead, comparisons of correlations between precursor and product FFAs among FEANS and control subjects were used to indirectly reflect a possible disruption within metabolic pathways.

Last of all, subjects in the AP group were in drug-free status at the time of enrollment. Patients in this group were recruited after the relapse without at least 30 days of treatment. Therefore, we cannot completely tease out the medication effect on the AP groups in terms of FFA levels. Moreover, low sample size and a lack of information regarding smoking status also became the limitations of the study.

\section{CONCLUSION}

Significantly reduced plasma FFAs levels were observed for FEANS patients, especially in 16:0,18:2n6c, 20:4n6, and total FFA levels, when compared with those levels in the (AP and HC) control groups. Also, disrupted correlations of fatty acids within saturated and n-6 fatty acid families were observed. 
These findings suggested an increased demand of membrane regeneration, a homeostatic imbalance of the fatty acid biosynthesis pathway, and a potential increase in beta oxidations.

\section{DATA AVAILABILITY STATEMENT}

The raw data supporting the conclusions of this article will be made available by the authors, without undue reservation, to any qualified researcher.

\section{ETHICS STATEMENT}

All participants provided written informed consent prior to participation in any research procedures. The study was approved by the Institutional Review Boards of the University of Pittsburgh and the VA Pittsburgh Healthcare System.

\section{AUTHOR CONTRIBUTIONS}

$\mathrm{XZ}$ and JY designed the research. XZ and TL performed the research. XZ, TL, GH, and HC wrote the manuscript. All authors contributed to the article and approved the submitted version.

\section{FUNDING}

This project was supported in part by the Merit Reviews 1I01CX000110 (JY) and Senior Research Career Scientist Award (JY) from the United States (U.S.) Department of Veterans Affairs

\section{REFERENCES}

Baumann, N., and Pham-Dinh, D. (2001). Biology of oligodendrocyte and myelin in the mammalian central nervous system. Physiol. Rev. 81, 871-927. doi: 10. 1152/physrev.2001.81.2.871

Benyó, Z., Gille, A., Kero, J., Csiky, M., Suchánková, M. C., Nüsing, R. M., et al. (2005). GPR109A (PUMA-G/HM74A) mediates nicotinic acid-induced flushing. J. Clin. Invest. 115, 3634-3640. doi: 10.1172/JCI23626

Bitanihirwe, B. K., and Woo, T. U. W. (2011). Oxidative stress in schizophrenia: an integrated approach. Neurosci. Biobehav. Rev. 35, 878-893. doi: 10.1016/j. neubiorev.2010.10.008

Boskovic, M., Vovk, T., Kores Plesnicar, B., and Grabnar, I. (2011). Oxidative stress in schizophrenia. Curr. Neuropharmacol. 9, 301-312. doi: 10.2174/ 157015911795596595

Brisch, R., Saniotis, A., Wolf, R., Bielau, H., Bernstein, H.-G., Steiner, J., et al. (2014). The Role of dopamine in schizophrenia from a neurobiological and evolutionary perspective: old fashioned, but still in vogue. Front. Psychiatry 5:47. doi: $10.3389 /$ fpsyt.2014.00047

Casey, D. E., Haupt, D. W., Newcomer, J. W., Henderson, D. C., Sernyak, M. J., Davidson, M., et al. (2004). Antipsychotic-induced weight gain and metabolic abnormalities: implications for increased mortality in patients with schizophrenia. J. Clin. Psychiatry 65, 4-18.

Chrast, R., Saher, G., Nave, K. A., and Verheijen, M. H. (2010). Lipid metabolism in myelinating glial cells: lessons from human inherited disorders and mouse models. J. Lipid Res. 52, 419-434. doi: 10.1194/jlr.R009761

Dennis, E. A. (1994). Diversity of group types, regulation, and function of phospholipase A2. J. Biol. Chem. 269, 13057-13060.
Biomedical Laboratory Research and Development Service (JY), infrastructure supported from the Veterans Affairs VISN 4 Mental Illness Research, Education, and Clinical Center, and supported from the National Institutes of Health [MH58141, R21 MH102565, and R03 MH070434 (JY); MH64023 and K02 MH01180 (Matcheri Keishavan, MD); MH45156 (David Lewis, MD, Director); and NIH/NCRR/GCRC M01 RR00056 (Arthur Levine, MD)], Hunan Provincial Natural Science Foundation of China [2017JJ3444 (HC)], National Natural Science Foundation of China [NSFC81401113 (HC)], Wu Jieping Medical Foundation Funded Special Clinical Research Project [320.6750.2020-04-2 (HC)], and the Fundamental Research Funds for the Central Universities of Central South University [2019zzts1049 and 2020zzts884 (HC)].

\section{ACKNOWLEDGMENTS}

We would like to thank Dr. Debra Montrose, Dr. Ravinder Reddy, and Dr. Matcheri Keshavan for the recruitment of patients and leadership in the collection of clinical data for this study. The views expressed in this article are those of the authors and do not necessarily represent the position or policy of the Department of Veterans Affairs or the United States Government.

\section{SUPPLEMENTARY MATERIAL}

The Supplementary Material for this article can be found online at: https://www.frontiersin.org/articles/10.3389/fnins. 2020.00784/full\#supplementary-material

Dietrich-Muszalska, A., and Kontek, B. (2010). Lipid peroxidation in patients with schizophrenia. Psychiatry Clin. Neurosci. 64, 469-475. doi: 10.1111/j.1440-1819. 2010.02132.x

Flower, R. J., and Blackwell, G. J. (1976). The importance of phospholipase-A2 in prostaglandin biosynthesis. Biochem. Pharmacol. 25, 285-291. doi: 10.1016/ 0006-2952(76)90216-1

Gattaz, W. F., and Brunner, J. (1996). Phospholipase A2 and the hypofrontality hypothesis of schizophrenia. Prostaglandins Leukotrienes Essential Fatty Acids 55, 109-113. doi: 10.1016/S0952-3278(96)90154-4

Gattaz, W. F., Hübner, C. V., Nevalainen, T. J., Thuren, T., and Kinnunen, P. K. (1990). Increased serum phospholipase A2 activity in schizophrenia: a replication study. Biol. Psychiatry 28, 495-501.

GBD 2017 Disease and Injury Incidence and Prevalence Collaborators, (2018). Global, regional, and national incidence, prevalence, and years lived with disability for 354 diseases and injuries for 195 countries and territories, 19902017: a systematic analysis for the Global Burden of Disease Study 2017. Lancet 392, 1789-1858. doi: 10.1016/S0140-6736(18)32279-7

Holman, R. T., Smythe, L., and Johnson, S. (1979). Effect of sex and age on fatty acid composition of human serum lipids. Am. J. Clin. Nutr. 32, 2390-2039. doi: $10.1093 /$ ajen/32.12.2390

Horrobin, D. F., Manku, M. S., Hillman, H., Iain, A., and Glen, M. (1991). Fatty acid levels in the brains of schizophrenics and normal controls. Biol. Psychiatry 30, 795-805. doi: 10.1016/0006-3223(91)90235-e

Karam, C. S., Ballon, J. S., Bivens, N. M., Freyberg, Z., Girgis, R. R., Lizardi-Ortiz, J. E., et al. (2010). Signaling Pathways in Schizophrenia: emerging targets and therapeutic strategies. Trends Pharmacol. Sci. 31, 381-390. doi: 10.1016/j.tips. 2010.05.004 
Kerner, B. (2009). Glutamate Neurotransmission in Psychotic Disorders and Substance Abuse. Open Psychiatry J. 3, 1-8. doi: 10.2174/1874354400903010001

Kubicki, M., McCarley, R. W., and Shenton, M. E. (2005). Evidence for white matter abnormalities in schizophrenia. Curr. Opin. Psychiatry 18, 121-134. doi: 10.1097/00001504-200503000-00004

Kuehl, F. A., and Egan, R. W. (1980). Prostaglandins, arachidonic acid, and inflammation. Science 210, 978-984. doi: 10.1126/science.6254151

Lai, E., De Lepeleire, I., Crumley, T. A., Liu, F., Wenning, L. A., Michiels, N., et al. (2007). Suppression of niacin-induced vasodilation with an antagonist to prostaglandin D2 receptor subtype 1. Clin. Pharmacol. Therapeut. 81, 849-857. doi: $10.1038 /$ sj.clpt.6100180

Lener, M. S., Wong, E., Tang, C. Y., Byne, W., Goldstein, K. E., Blair, N. J., et al. (2014). White matter abnormalities in schizophrenia and schizotypal personality disorder. Schizophrenia Bull. 41, 300-310. doi: 10.1093/schbul/ sbu093

Lepage, G., and Roy, C. C. (1988). Specific methylation of plasma nonesterified fatty acids in a one-step reaction. J. Lipid Res. 29, 227-235.

Maciejewski-Lenoir, D., Richman, J. G., Hakak, Y., Gaidarov, I., Behan, D. P., and Connolly, D. T. (2006). Langerhans cells release prostaglandin D2 in response to nicotinic acid. J. Invest. Dermatol. 126, 2637-2646. doi: 10.1038/sj.jid.5700586

Manku, M. S., Horrobin, D. F., Huang, Y. S., and Morse, N. (1983). Fatty acids in plasma and red cell membranes in normal humans. Lipids 18, 906-908. doi: 10.1007/BF02534572

McEvoy, J., Baillie, R. A., Zhu, H., Buckley, P., Keshavan, M. S., Nasrallah, H. A., et al. (2013). Lipidomics reveals early metabolic changes in subjects with schizophrenia: effects of atypical antipsychotics. PLoS One 8:e68717. doi: 10. 1371/journal.pone.0068717

Messamore, E., Hoffman, W. F., and Yao, J. K. (2010). Niacin sensitivity and the arachidonic acid pathway in schizophrenia. Schizophr. Res. 122, 248-256. doi: 10.1016/j.schres.2010.03.025

Messamore, E., and Yao, J. K. (2016). Phospholipid, arachidonate and eicosanoid signaling in schizophrenia. OCL 23:D112. doi: 10.1051/ocl/2015054

Misiak, B., Stanczykiewicz, B., Laczmanski, L., and Frydecka, D. (2017). Lipid profile disturbances in antipsychotic-naive patients with first-episode nonaffective psychosis: a systematic review and meta-analysis. Schizophr. Res. 190, 18-27. doi: 10.1016/j.schres.2017.03.031

Morrow, J. D., Awad, J. A., Oates, J. A., and Roberts, L. J. II (1992). Identification of skin as a major site on prostaglandin D2 release following oral administration of niacin in humans. J. Invest. Dermatol. 98, 812-815. doi: 10.1111/1523-1747. ep12499963

Morrow, J. D., Parsons, W. G. III, and Roberts, L. J. II (1989). Release of markedly increased quantities of prostaglandin D2 in vivo in humans following the administration of nicotinic acid. Prostaglandins 38, 263-274. doi: 10.1016/ 0090-6980(89)90088-9

Noponen, M., Sanfilipo, M., Samanich, K., Ryer, H., Ko, G., Angrist, B., et al. (1993). Elevated PLA2 activity in schizophrenics and other psychiatric patients. Biol. Psychiatry 34, 641-649. doi: 10.1016/0006-3223(93)90157-9

Peters, B. D., Machielsen, M. W., Hoen, W. P., Caan, M. W., Malhotra, A. K., Szeszko, P. R., et al. (2012). Polyunsaturated fatty acid concentration predicts myelin integrity in early-phase psychosis. Schizophr. Bull. 39, 830-838. doi: $10.1093 /$ schbul/sbs089

Pettegrew, J. W., Keshavan, M. S., Panchalingam, K., Strychor, S., Kaplan, D. B., Tretta, M. G., et al. (1991). Alterations in brain high-energy phosphate and membrane phospholipid metabolism in first-episode, drugnaive schizophrenics: a pilot study of the dorsal prefrontal cortex by in vivo phosphorus 31 nuclear magnetic resonance spectroscopy. Arch. Gen. Psychiatry $48,563-568$.

Prabakaran, S., Swatton, J. E., Ryan, M. M., Huffaker, S. J., Huang, J. J., Griffin, J. L., et al. (2004). Mitochondrial dysfunction in schizophrenia: evidence for compromised brain metabolism and oxidative stress. Mol. Psychiatry 9:684.

Puri, B. K., Easton, T., Das, I., Kidane, L., and Richardson, A. J. (2001). The niacin skin flush test in schizophrenia: a replication study. Int. J. Clin. Pract. $55,368-370$.

Rapoport, S. I. (2001). In vivo fatty acid incorporation into brain phosholipids in relation to plasma availability, signal transduction and membrane remodeling. J. Mol. Neurosci. 16, 243-261. doi: 10.1385/JMN:16:23:243

Reddy, R. D., Keshavan, M. S., and Yao, J. K. (2004). Reduced red blood cell membrane essential polyunsaturated fatty acids in first episode schizophrenia at neuroleptic-naive baseline. Schizophr. Bull. 30, 901-911. doi: 10.1093/ oxfordjournals.schbul.a007140

Ross, B. M., Hudson, C., Erlich, J., Warsh, J. J., and Kish, S. J. (1997). Increased phospholipid breakdown in schizophrenia: evidence for the involvement of a calcium-independent phospholipase A2. Arch. Gen. Psychiatry 54, 487-494. doi: 10.1001/archpsyc.1997.01830170113015

Šakić, M., Karlović, D., Vidrih, B., Peitl, V., Crnković, D., and Vrkić, N. (2016). Increased calcium-independent lipoprotein phospholipase A2 but not protein S100 in patients with schizophrenia. Psychiatr. Danubina 28, 0-50.

Schaeffer, E. L., Bassi, F., and Gattaz, W. F. (2005). Inhibition of phospholipase A2 activity reduces membrane fluidity in rat hippocampus. J. Neural. Transm. 112, 641-647. doi: 10.1007/s00702-005-0301-9

Schmitt, A., Wilczek, K., Blennow, K., Maras, A., Jatzko, A., Petroianu, G., et al. (2004). Altered thalamic membrane phospholipids in schizophrenia: a postmortem study. Biol. Psychiatry 56, 41-45. doi: 10.1016/j.biopsych.2004.03.019

Sears, B., and Perry, M. (2015). The role of fatty acids in insulin resistance. Lipids Health Dis. 14:121. doi: 10.1186/s12944-015-0123-121

Smesny, S., Kinder, D., Willhardt, I., Rosburg, T., Lasch, J., Berger, G., et al. (2005). Increased calcium-independent phospholipase A2 activity in first but not in multiepisode chronic schizophrenia. Biol. Psychiatry 57, 399-405. doi: 10.1016/ j.biopsych.2004.11.018

St-Gelais, F., Ménard, C., Congar, P., Trudeau, L. E., and Massicotte, G. (2004). Postsynaptic injection of calcium-independent phospholipase A2 inhibitors selectively increases AMPA receptor-mediated synaptic transmission. Hippocampus 14, 319-325. doi: 10.1002/hipo. 10176

Stoll, L. L., and Spector, A. A. (1984). Changes in serum influence the fatty acid composition of established cell lines. In Vitro 20, 732-738. doi: 10.1007/ BF02618879

Taha, A. Y., Cheon, Y., Ma, K., Rapoport, S. I., and Rao, J. S. (2013). Altered fatty acid concentrations in prefrontal cortex of schizophrenic patients. J. Psychiatr. Res. 47, 636-643. doi: 10.1016/j.jpsychires.2013.01.016

Tang, Y., Zhou, L., Gunnet, J. W., Wines, P. G., Cryan, E. V., and Demarest, K. T. (2006). Enhancement of arachidonic acid signaling pathway by nicotinic acid receptor HM74A. Biochem. Biophys. Res. Commun. 345, 29-37. doi: 10.1016/j. bbrc.2006.04.051

Van der Kemp, W. J. M., Klomp, D. W. J., Kahn, R. S., Luijten, P. R., and Pol, H. H. (2012). A meta-analysis of the polyunsaturated fatty acid composition of erythrocyte membranes in schizophrenia. Schizophr. Res. 141, 153-161. doi: 10.1016/j.schres.2012.08.014

Von Schacky, C., and Weber, P. C. (1985). Metabolism and effects on platelet function of the purified eicosapentaenoic and docosahexaenoic acids in humans. J. Clin. Invest. 76, 2446-2450. doi: 10.1172/JCI112261

Walsh, T., McClellan, J. M., McCarthy, S. E., Addington, A. M., Pierce, S. B., Cooper, G. M., et al. (2008). Rare structural variants disrupt multiple genes in neurodevelopmental pathways in schizophrenia. Science 320, 539-543. doi: 10.1126/science. 1155174

Ward, P. E., Sutherland, J., Glen, E. M. T., and Glen, A. I. M. (1998). Niacin skin flush in schizophrenia: a preliminary report. Schizophr. Res. 29, 269-274. doi: 10.1016/s0920-9964(97)00100-x

Weinberger, D. R., Berman, K. F., and Illowsky, B. P. (1988). Physiological dysfunction of dorsolateral prefrontal cortex in schizophrenia: III. A new cohort and evidence for a monoaminergic mechanism. Arch. Gen. Psychiatry $45,609-615$.

Weinberger, D. R., Berman, K. F., Suddath, R., and Torrey, E. F. (1992). Evidence of dysfunction of a prefrontal-limbic network in schizophrenia: a magnetic resonance imaging and regional cerebral blood flow study of discordant monozygotic twins. Am. J. Psychiatry 149, 890-897. doi: 10.1176/ajp.149.7.890

Wu, R. R., Zhao, J. P., Liu, Z. N., Zhai, J. G., Guo, X. F., Guo, W. B., et al. (2006). Effects of typical and atypical antipsychotics on glucose-insulin homeostasis and lipid metabolism in first-episode schizophrenia. Psychopharmacology 186, 572-578. doi: 10.1007/s00213-006-0384-5

Xu, F., Fan, W., Wang, W., Tang, W., Yang, F., Zhang, Y., et al. (2019). Effects of omega-3 fatty acids on metabolic syndrome in patients with schizophrenia: a 12-week randomized placebo-controlled trial. Psychopharmacology 236, 12731279. doi: 10.1007/s00213-018-5136-9

Yang, X., Sun, L., Zhao, A., Hu, X., Qing, Y., Jiang, J., et al. (2017). Serum fatty acid patterns in patients with schizophrenia: a targeted 
metabonomics study. Transl. Psychiatry $7:$ e1176. doi: 10.1038/tp.20 17.152

Yao, J. K., Stanley, J. A., Reddy, R. D., Keshavan, M. S., and Pettegrew, J. W. (2002). Correlations between peripheral polyunsaturated fatty acid content and in vivo membrane phospholipid metabolites. Biol. Psychiatry 52, 823-830. doi: 10.1016/S0006-3223(02)01397-5

Yehuda, S., Rabinovitz, S., Carasso, R. L., and Mostofsky, D. I. (2002). The role of polyunsaturated fatty acids in restoring the aging neuronal membrane. Neurobiol. Aging 23, 843-853. doi: 10.1016/S0197-4580(02) 00074-X

Zhou, X., Reddy, R., Montrose, D., Haas, G., Keshavan, M., and Yao, J. (2018). S218. A Potential Therapeutic Target of Plasma Free Fatty Acids in First-Episode Antipsychotic-Naïve Patients With Schizophrenia. Biol. Psychiatry 83:S432. doi: 10.1016/j.biopsych.2018.02.1110
Zhou, X., Reddy, R., Montrose, D., Haas, G., Keshavan, M. S., and Yao, J. (2017). A potential role of plasma free fatty acids in schizophrenia pathology. Schizophr. Bull. 43:S12. doi: 10.1093/schbul/sbx021.033

Conflict of Interest: The authors declare that the research was conducted in the absence of any commercial or financial relationships that could be construed as a potential conflict of interest.

Copyright ( 2020 Zhou, Long, Haas, Cai and Yao. This is an open-access article distributed under the terms of the Creative Commons Attribution License (CC BY). The use, distribution or reproduction in other forums is permitted, provided the original author(s) and the copyright owner(s) are credited and that the original publication in this journal is cited, in accordance with accepted academic practice. No use, distribution or reproduction is permitted which does not comply with these terms. 